\title{
Metastasis Targeted Therapies in Renal Cell Cancer
}

\author{
(1) K. Fehmi Narter MD', (1) Bora Özveren MD² \\ 'Acıbadem Mehmet Ali Aydınlar University, Kadıköy Hospital, Clinic of Urology, Istanbul, Turkey \\ ${ }^{2}$ Acibadem Mehmet Ali Aydinlar University Faculty of Medicine, Department of Urology, Istanbul, Turkey
}

\begin{abstract}
Metastatic renal cell cancer is a malignant disease and its treatment has been not been described clearly yet. These patients are generally symptomatic and resistant to current treatment modalities. Radiotherapy, chemotherapy, and hormonal therapy are not curative in many of these patients. A multimodal approach consisting of cytoreductive nephrectomy, systemic therapy (immunotherapy or targeted molecules), and metastasectomy has been shown to be hopeful in prolonging the survival and improving the quality of life in some of these patients. Patients with oligometastatic disease and good performance status have better results following this multimodal approach. Cytoreductive nephrectomy and adjuvant/neoadjuvant systemic therapies (immunotherapy, targeted therapy) have been investigated for treatment options of metastatic renal cancer patients. After better understanding of the genetic basis and the molecular biology of the renal cell carcinoma, targeted molecular therapies and immunotherapies have emerged as more efficient alternative therapy options with moderate adverse effects. Metastasectomy in some of these patients improves survival and quality of life, especially in those with lung and bone metastases. In this review we will summarize treatment options for metastatic renal cancer patients.
\end{abstract}

Keywords: Cytoreductive nephrectomy, immunotherapy, metastasectomy, metastatic renal cancer, targeted molecular therapy

\section{Introduction}

Renal cell carcinoma (RCC) is a highly vascularized cancer originating in the proximal tubule of the renal cortex, and accounts for approximately $85 \%$ of all renal masses. Despite the classic clinical triad of macroscopic hematuria, mass, and side pain, about half of all cases are detected incidentally during imaging. It is more common in males and accounts for about $2-3 \%$ of all cancers. The rate of locally advanced disease is approximately $20-25 \%$, and metastasis is common due to its hypervascular structure resulting from the molecular mechanisms involved in its etiology [von Hippel-Lindau, hypoxia-inducible factor, vascular endothelial growth factor (VEGF)] due to the poor response of metastatic RCC (mRCC) to chemotherapy and radiotherapy, the development and use of current treatments have only slightly increased overall survival (OS) rates from 5 months to 15 months. Metastasis can occur in a wide variety of sites, commonly affecting the lungs, bones, distant lymph nodes, liver, and brain. In approximately $25-30 \%$ of RCC patients, the primary tumor has metastasized despite appearing limited to the kidney in nephrectomy (1). mRCC is associated with very high mortality; the average 2-year OS rate is only $10-20 \%$, with average survival of 10 months (2).

Immunotherapy and target-specific agents developed based on a clear understanding of the underlying molecular mechanisms of mRCC have offered slight survival advantages. However, it is important to consider that this limited increase in survival may be related to the fact that these treatments have been trialed and used in patients with more advanced and even metastatic disease compared to conventional and established treatment alternatives such as surgery, radiotherapy, or chemotherapy. In addition to these current treatments, the roles of cytoreductive nephrectomy (CN) and metastasectomy in the treatment of $\mathrm{mRCC}$ are areas of intensive research. In this review, targeted therapy/immunotherapy options for mRCC patients, the contribution of $\mathrm{CN}$, and metastasectomy methods will be summarized in light of the current literature.

\section{Targeted Therapies/Immunotherapy}

In place of immunotherapy containing interferon (IFN) and interleukin (IL)-2, which have more side effects, the less toxic VEGF and target of rapamycin kinase (mTOR) suppressive therapeutic agents are currently favored. The order of use and combinations of agents constitute an important area of research in adjuvant/neoadjuvant targeted therapy/immunotherapy studies. Prior to FDA approval of sorafenib and sunitinib as first-line treatments in 2005, the toxic high-dose IL-2 provided long-term remission in approximately $10 \%$ of patients, though 
its partial response rate was reported to be slightly better. Moreover, during patient selection, ideal candidates were identified as those with good performance status who had no bone metastasis, low volume tumor, and prior nephrectomy. The first-line treatments sunitinib and pazopanib are orally administered drugs with multiple targets such as VEGF receptors, platelet-derived growth factor receptors (PDGF), and other tyrosine kinases. As first generation molecules, sunitinib and pazopanib have been found to elicit higher response rates and longer progression-free survival (PFS) rate than both placebo and IFN-alpha, and OS has been reported as 30 months $(3,4)$. Motzer et al. (3) compared sunitinib and IFN in the treatment of $\mathrm{mRCC}$, and reported their respective PFS rates as 11 and 5 months and response rates as $31 \%$ and $6 \%$. Escudier et al. (5) compared sorafenib and placebo in patients who did not respond to immunotherapy and radiotherapy, and reported PFS as 5.5 months vs. 2.8 months, respectively. Although sunitinib and pazopanib were found to be equally effective in the COMPARZ study, pazopanib was more advantageous in terms of side effects and quality of life (6). Hudes et al. (7) compared IFN with weekly administered temsirolimus in $\mathrm{mRCC}$ patients with poor prognosis and reported longer OS [hazard ratio (HR) 0.73 ] and PFS (5.5 vs. 3.1 months) in the temsirolimus group. Combined therapy has not been shown to be superior to temsirolimus alone. It was reported that it may be appropriate as first-line treatment in low-risk RCC. Metabolic toxic effects (hyperglycemia, hyperlipidemia, hypercholesterolemia) are side effects of this class of agents.

Axitinib is an orally administered VEGF inhibitor. A phase 3 trial comparing axitinib and sorafenib as first-line treatment demonstrated adequate safety and efficacy of axitinib and reported that it could be used as a first-line drug (8). It has also been reported that administering bevacizumab in combination with IFN-alpha as first-line treatment provides a higher response rate and longer PFS compared to IFN-alpha alone (9).

Patients whose disease progresses under treatment with a VEGF receptor (VEGFR)-targeted agent can be switched to another VEGFR-targeted agent or an mTOR inhibitor. According to phase 3 trial results, everolimus and axitinib can be used as second-line therapy after first-line VEGF-targeted therapy (10). Everolimus is an orally administered mTOR suppressant and is not recommended as first-line therapy. Placebo-controlled randomized phase 3 trials have shown that everolimus extends PFS in patients who exhibited progression during sunitinib/sorafenib treatment (11). Resistance may develop against VEGF or mTOR-targeted therapies over time. Combined use of VEGF and mTOR-targeted suppressants may delay resistance. Randomized trials comparing the combination of bevacizumab with temsirolimus or everolimus to bevacizumab and IFN-alpha alone showed that combined therapy did not increase efficacy, but resulted in higher rates of drug-induced toxic effects $(12,13)$. In a randomized phase 2 trial, it is reported that the combination of everolimus and lenvatinib [dual VEGFR/fibroblast growth factor receptor (FGFR) inhibitor] is superior to everolimus alone in terms of PFS and OS (14). In combination therapies, a lower starting dose of each agent is necessary due to increased toxicity. The likely mechanism of resistance involves the tyrosine kinases FGFR, MET, and AXL in an alternative non-VEGF pathway (15). In a phase 3 trial, treatment with cabozantinib, an inhibitor of VEGF receptor, MET, and AXL, increased PFS and OS more than standard everolimus therapy in patients who developed resistance to first-line VEGF-targeted therapy (16).

Vaccines and the targeting of cytotoxic T-lymphocyte associated protein 4 (CTLA-4), programmed cell death protein 1 (PD1 ), and programmed cell death protein 1 ligand (PD-L1) as immune checkpoint inhibitors have opened new horizons in immunotherapy. Nivolumab is a fully human monoclonal immunoglobulin G4 antibody specific to PD-1. According to preliminary results, this checkpoint inhibitor elicited a better response compared to everolimus in patients showing progression with VEGF-targeted agents, and also resulted in fewer side effects and improved quality of life (17).

In brief, five antiangiogenic agents (pazopanib, axitinib, bevacizumab, cabozantinib, and lenvatinib), as well as the mTOR inhibitors temsirolimus and everolimus and the immune checkpoint suppressant nivolumab were approved by the FDA after sorafenib and sunitinib. Therapies should be selected according to individual factors, side effects, and comorbidities (caution should be exercised when using mTOR inhibitors in patients with diabetes mellitus or nivolumab in patients with autoimmune diseases).

The treatment of RCC with non-clear cell histopathology is the same as that of RCC, despite having different molecular properties. According to the National Comprehensive Cancer Network guidelines, these are rare cases and the chances of success with systemic therapy are low. In the literature, it is reported that sunitinib extends PFS more than everolimus in non-clear cell RCC, especially the papillary type (18). Limited response to chemotherapy was obtained with a doxorubicin and gemcitabine combination in sarcomatoid tumors and with a combination of platinum-based drugs in collecting duct carcinomas.

Various plasma, tissue, and tumor biomarkers are being studied to improve prediction and efficacy in targeted therapies. Further studies are needed to determine the predictive value of mTOR pathway genes TSC $1 / 2$ and mTOR genes in the prediction of the efficacy of these inhibitors, and the utility of high pretreatment PD-L1 expression levels as a predictive marker in nivolumab therapy. Intratumoral heterogeneity is an important problem in determining this type of marker.

A randomized phase 2 study demonstrated the superiority of cabozantinib to standard first-line sunitinib in moderateand low-risk patients (19). Combination treatments such as lenvatinib with everolimus or nivolumab may be considered in cabozantinib-resistant cases. Currently, nivolumab and lowdose ipilimumab (a checkpoint suppressant that inhibits CTLA4) are being compared with sunitinib. The treatment decision algorithm recommended in $\mathrm{mRCC}$ is summarized in Figure 1. Although RCCs are known to be radiotherapy-resistant tumors, radiotherapy may be beneficial for the palliation of symptoms, especially in select cases with bone or brain metastasis. In patients with malignant and symptomatic bone lesions, it has been suggested that stereotactic ablative radiotherapy and bisphosphonate use may provide metastatic local control. In cases of brain metastasis, surgery, stereotactic radiosurgery 
(STRS), or whole-brain radiotherapy may be preferred as other alternatives prior to systemic therapy.

Commonly used risk assessment methods are the Memorial Sloan-Kettering Cancer Center model (lactate dehydrogenase, corrected calcium, serum hemoglobin, Karnofsky performance status, and time from diagnosis to start of treatment) and the International Metastatic Renal Cell Carcinoma Database Consortium model (IMDC) (Heng Criteria) (low hemoglobin, high calcium, $\leq 80 \%$ Karnofsky score, $<1$ year between diagnosis and initiation of systemic treatment, high neutrophil count, and high platelet count). Differences in systemic or local progression time lead to indecision regarding the application of medical or surgical treatment. Aggressive surgical resection of metastatic foci may not only be palliative, but may also provide longterm remission or cure. These criteria can be used in decisions regarding metastasectomy and first-line therapy. Ongoing studies of combination therapies in $\mathrm{MRCC}$ and these agents' mechanisms of action are summarized in Tables 1 and 2.

\section{Metastasectomy}

Metastasectomy can be performed at the same time as nephrectomy to ensure disease-free survival, upon the development of post-nephrectomy recurrence, or after systemic therapy following nephrectomy. It is shown to improve diseasespecific survival (DSS) in patients with good overall performance status, with a low volume or number of metastases (solitary is optimal), and with metastasis limited to one organ (adrenal, lungs, bones). A positive response to immunotherapy has been reported to reduce tumor burden and associated metastatic disease by $20-30 \%$ and extend PFS or OS. For metastases of the brain and bones it is done as a palliative procedure. In oligometastatic disease, it is of greatest benefit to patients who have long disease-free intervals and are able to undergo full surgical resection. Currently, there is no randomized study comparing metastasectomy with medical treatment. Despite a lack of high-quality evidence, it is reported that metastasectomy can improve outcomes in selected cases. In a large-scale study in which $28 \%$ of patients with mRCC underwent metastasectomy, survival time was 44.3 months among patients who underwent metastasectomy and 16.4 months among those who did not (21). In another series of 278 patients, Kavolius et al. (22) compared patients who underwent curative metastasectomy at the first recurrence with those who underwent noncurative surgery or were treated nonsurgically. Five-year OS was 44\%, 14\%, and 11\%, respectively (22). They obtained the best results in patients with solitary lung metastasis. Positive predictive factors include metastasis in a single location at first recurrence, the number of metastases ( $\leq 3$ foci), complete curative resectability of metastases, long disease-free interval, metachronous recurrence, and good performance status. In a recent systematic review, groups that underwent complete metastasectomy were compared with groups that underwent incomplete metastasectomy or did not undergo metastasectomy. Complete resection was associated with significant increases in DSS and OS (40.8 months vs. 14.8 months). HRs for DSS and OS indicated improved survival with complete resection regardless of organ location (23).

Combination of systemic therapy and metastasectomy: Data concerning the combination of targeted therapy and

\begin{tabular}{|c|c|}
\hline Treatment & Study \\
\hline $\begin{array}{l}\text { Pembrolizumab-lenvatinib vs } \\
\text { everolimus-lenvatinib vs sunitinib }\end{array}$ & CLEAR \\
\hline Nivolumab-ipilimumab vs sunitinib & CheckMate 214 \\
\hline Atezolizumab-bevacizumab vs sunitinib & IMmotion151 \\
\hline Avelumab-axitinib vs sunitinib & JAVELIN Renal 101 \\
\hline Pembrolizumab-axitinib vs sunitinib & KEYNOTE-426 \\
\hline $\begin{array}{l}\text { Autologous dendritic cell } \\
\text { immunotherapy-sunitinib vs sunitinib }\end{array}$ & ADAPT \\
\hline
\end{tabular}

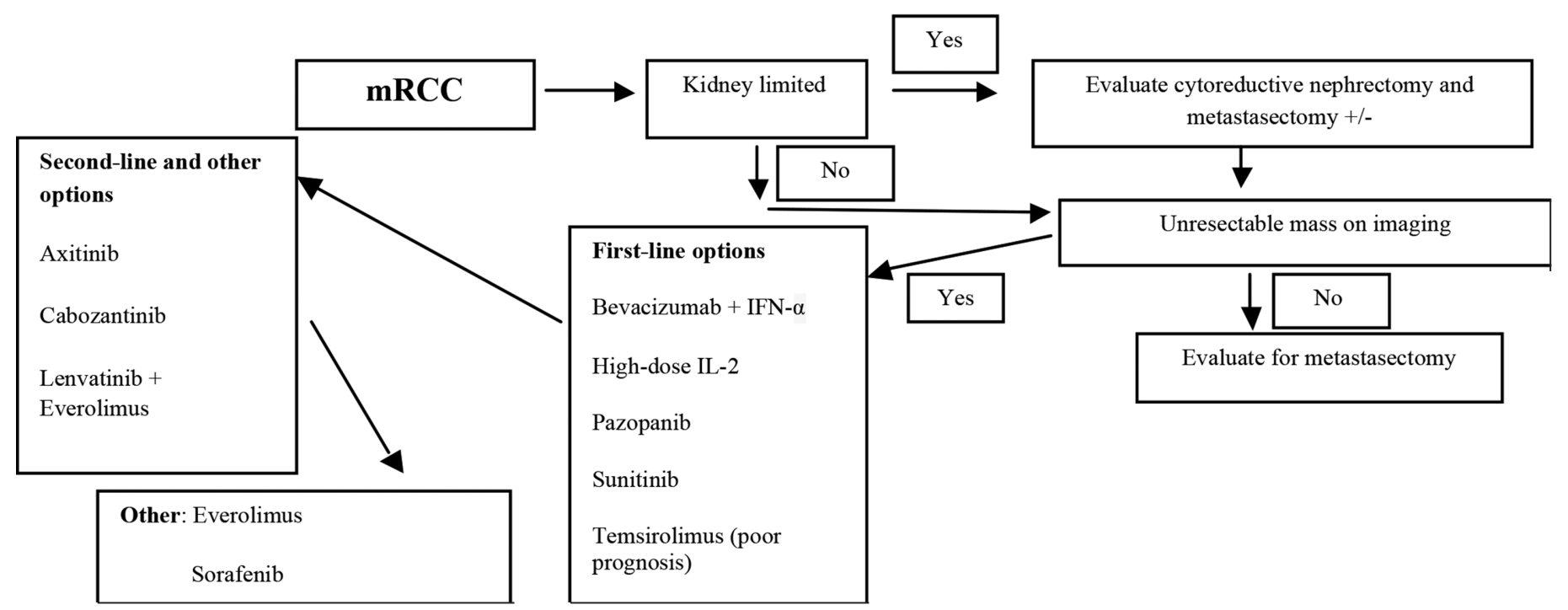

Figure 1. Recommended treatment decision algorithm for metastatic renal cell carcinoma mRCC: Metastatic renal cell carcinoma, IL-2: Interleukin-2, IFN: Interferon 
metastasectomy are extremely limited. The diversity of drugs and heterogeneity in treatment initiation times in the immunotherapy era present serious challenges in analysis. Karam et al. (24) assessed metastasectomy in 22 patients who had previously undergone at least one cycle of targeted therapy as pseudo-neoadjuvant therapy. All detectable masses were removed, $50 \%$ of patients survived disease-free, and the other 11 patients (50\%) survived without the need for postoperative targeted therapy. Their study showed that targeted agents and metastasectomy provided long-term tumor-free survival in carefully selected patients (24). Prognostic markers can also be used for risk classification before metastasis surgery such as $\mathrm{CN}$. Low-risk patients are more suitable for metastasectomy. Different studies have identified various influential factors, including resectability, disease-free interval, number of metastases, pleural infiltration, synchronous presence of primary RCC and pulmonary metastasis, metastasis $>3 \mathrm{~cm}$, presence of a histologically proven mediastinal and/or hilar lymph node.

Wedge resection, segmentectomy, lobectomy, and pneumonectomy can be performed for a solitary lung metastasis in selected cases. Bone metastasis is associated with shorter survival. Metastases in the bones have been associated with substantial bone pain, spinal cord compression with neurologic deficits, pathologic fractures, and/or hypercalcemia (inadequate effect of targeted therapies on the bones). Treatment approaches used in such cases are curettage and cementation and/ or internal fixation, complete resection, and closed nailing procedures. Bone metastases are most commonly detected in the femur, humerus, and pelvis. Radiotherapy to the metastatic site can be applied after surgery in these patients. In addition to palliative pain relief, surgery may be recommended for solitary bone metastases to prevent pathologic fractures or spinal cord compression.

Because liver metastasis is an unfavorable indicator of disseminated disease, liver resection is less commonly reported in the literature. A solitary metastasis below $0.5 \mathrm{~cm}$ in size does not significantly affect survival. Patients with synchronous metastasis reportedly benefit less from surgery. Retroperitoneal recurrence (RPR) includes pathologically proven ipsilateral soft

\begin{tabular}{|l|l|}
\hline \multicolumn{2}{|l|}{$\begin{array}{l}\text { Table 2. Targeted agents used in metastatic renal cell carcinoma } \\
\text { and their mechanisms of action }\end{array}$} \\
\hline Targeted therapy agent & Inhibited pathway \\
\hline Bevacizumab & VEGF \\
\hline Axitinib & VEGFR, PDGFR \\
\hline Pazopanib & VEGFR, PDGFR \\
\hline Sunitinib & VEGFR, PDGFR \\
\hline Sorafenib & VEGFR, PDGFR \\
\hline Cabozantinib & C-MET, AXL, VEGFR \\
\hline Lenvatinib & FGFR, VEGFR \\
\hline Everolimus & mTOR \\
\hline Temsirolimus & mTOR \\
\hline Nivolumab & PD1-PDL1 \\
\hline $\begin{array}{l}\text { VEGF: Vascular endothelial growth factor, VEGFR: Vascular endothelial growth } \\
\text { factor receptor, PDGFR: Platelet-derived } \\
\text { Fibroblast growth factor receptor, mTOR: Mechanistic target of rapamycin, PD1: } \\
\text { Programmed cell death protein 1, PDL1: Programmed cell death protein 1 ligand }\end{array}$ \\
\hline
\end{tabular}

tissue/psoas, ipsilateral lymph node, and ipsilateral adrenal involvement. Location of RPR (renal fossa/soft tissue, lymph node, or adrenal tissue) does not seem to affect DSS. Although not supported by sufficient evidence, aggressive resection of RPR may be curative in select patients.

Patients with brain metastasis have poor prognosis, with an average survival time of 4-11 months and a 5-year survival rate of $12 \%$. A palliative approach is usually taken. Although central nervous system lesions may be asymptomatic, they may lead to loss of function, headache associated with edema, neuropathy, and sensory or motor loss over time. Treatment options include whole-brain/conventional radiotherapy, STRS, or surgical resection. Radiosurgery or surgical resection improves survival in select patients. Ikushima et al. (25) compared brain metastasectomy followed by conventional radiotherapy with STRS or conventional radiotherapy alone, and reported median survival times of 18,25 , and 4 months, respectively. Initial number of tumors has been identified as an independent predictive factor for central nervous system recurrence. The role of tyrosine kinase inhibitors in the progression or remission of brain metastases has not yet been clearly defined. The low response rates in survival are partly due to the inability of targeted therapeutic agents to cross the blood-brain barrier.

At $31 \%$, the rate of concurrent thyroid and pancreatic metastases is high. Therefore, if one is detected, the other organ should also be investigated for metastasis. Table 3 shows the major organ metastases that occur in mRCC.

In brief, metastasectomy can improve survival in select cases as part of individualized treatment, and it can also be recommended for patients who do not respond to medical treatment alone. It should be kept in mind that in cases with isolated, resectable metastases, metastasectomy is of greatest benefit to those with long disease-free interval and good overall performance status.

\section{Cytoreductive Nephrectomy}

Surgery has an important role in $\mathrm{mRCC}$. The removal of a primary renal tumor in $\mathrm{mRCC}$ is referred to as CN. In two randomized phase 3 studies by the Southwest Oncology Group (SWOG) and the European Organisation for Research and Treatment of Cancer (EORTC), CN followed by IFN-alpha was shown to provide a significant survival advantage compared to treatment with IFN-alpha alone (11.1 months vs. 8.1 months and 17.0 months vs. 7.0 months, respectively) $(27,28)$. According to retrospective analysis of a large database, CN provided longer survival in those taking VEGF-targeted agents or mTOR inhibitors compared to the unoperated group (17.1 months vs. 7.7 months) (29). Patients with good performance status and low systemic disease burden are ideal candidates for $\mathrm{CN}$. The average interval between surgery and initiating IFN is 19 days. It is not clearly understood why CN improves OS. A primary tumor isolates immune cells and antibodies. Removal of the primary tumor is believed to be important in the treatment of RCC due to immune mechanisms which are associated with spontaneous regression of metastases. Nephrectomy allows these immune factors to act on metastases. RCC causes the release of VEGF, PDGF, FGF, and transforming growth factor beta. Removal of the primary tumor prevents the circulation of these growth hormones, thus reducing angiogenesis in the 
Narter and Özveren.

Treatment of Metastatic Renal Cancer

\begin{tabular}{|c|c|c|c|}
\hline Organ & Incidence (\%) & 5 -year overall survival (\%) & Characteristics favoring metastasectomy \\
\hline Lung & $45-75$ & $36-50$ & $\begin{array}{l}\text { Complete metastasectomy } \\
\text { Lung metastasis }(<7) \\
\text { Negative lymph node } \\
>23 \text { months RFS } \\
\text { Negative mediastinal lymph node } \\
\text { Resection if lymph nodes are present }\end{array}$ \\
\hline Liver & 20 & $18-43$ & $\begin{array}{l}\text { ECOG } 0 \\
\text { PNO in nephrectomy } \\
\text { Furhman } 1-2 \text { in nephrectomy } \\
\text { Metachronous metastasis at diagnosis } \\
\text { Solitary liver metastasis } \\
\text { No extrahepatic involvement }\end{array}$ \\
\hline Pancreas & $\leq 1$ & 72 & $\begin{array}{l}\text { Solitary metastasis } \\
\text { No extrapancreatic spread } \\
\text { No symptoms }\end{array}$ \\
\hline Thyroid & $\leq 1$ & 51 & $\begin{array}{l}\text { Solitary metastasis } \\
\text { Age }<70 \\
\text { No metastasis in the contralateral kidney }\end{array}$ \\
\hline
\end{tabular}

metastatic region.

Studies on the effect of immunomodulation on mRCC have established that response to IFN-alpha-2b and IL-2 does not exceed $15 \%$ in total. The addition of $\mathrm{CN}$ enabled reduction of total tumor burden and palliative symptomatic improvement (hematuria, pain, paraneoplastic symptoms such as anemia, hypercalciuria). This palliative improvement also increases tolerance to systemic therapies. However, it is also necessary to consider the perioperative morbidity and mortality of $\mathrm{CN}$ in difficult cases. In addition to publications indicating that $\mathrm{CN}$ is beneficial, there are also publications which suggest otherwise. It has been suggested that better results are obtained in patients who undergo primary nephrectomy and that metastases respond better to immunotherapy than primary tumors.

Although the SWOG and EORTC studies show that $\mathrm{CN}$ is beneficial in $\mathrm{mRCC}$, it is not yet clear which patients will benefit. Certain patient characteristics were identified in those studies which are favorable for CN: Removal of at least $75 \%$ of the tumor burden, absence of central nervous system, bone, or hepatic metastasis, adequate pulmonary and cardiac function, the Eastern Cooperative Oncology Group performance status of $0-1$, and dominant clear cell histology. Besides these factors, it has been reported that regional lymph node involvement, vital symptoms, solitary or multiple bone or lung metastasis, sarcomatoid features, and the thyroid-stimulating hormone level $>2 \mathrm{mIU} / \mathrm{L}$ are associated with poor prognosis. Other studies have identified various prognostic factors for survival: Low serum albumin level, high serum lactose dehydrogenase level, clinical stage T3/T4 tumor, presence of metastatic symptoms, presence of liver metastasis, blood transfusion, presence of a retroperitoneal or supradiaphragmatic lymph node at least 1 $\mathrm{cm}$ in size, age $\geq 60$ years, Afro-American race, tumor grade 3-4, primary tumor $>7 \mathrm{~cm}$, sarcomatoid histopathology, the presence of both visceral and distant node metastases, neutrophil/ lymphocyte ratio (above or below 4), and good performance status. They suggested that sarcopenia (nutritional status), low body mass index, low preoperative albumin level, and low preoperative hemoglobin level $(<3.5 \mathrm{~g} / \mathrm{dL})$ are poor prognostic factors. In patients undergoing $\mathrm{CN}$ followed by immunotherapy, immunotherapy response rates have been reported as $44 \%$ in cases of lung metastasis alone, $22 \%$ in cases of bone metastasis, and $14 \%$ for metastases in multiple locations. The number and location of distant metastases have been identified as an important prognostic factor for DSS. Lymph node involvement is a negative prognostic factor for survival compared with local disease. The contribution of immunotherapy to survival in patients with positive lymph nodes has not been determined. Trinh et al. (30) used the Surveillance, Epidemiology, and End Results database to examine 1415 patients who underwent CN, 619 of whom had nodal disease. Median DSS was reported as 7 months in cases with lymph node involvement, and OS rates in patients with and without nodal disease were $40.2 \%$ and $65.8 \%$ at 1 year. At 5 years, these figures fell to $11.5 \%$ and $24.8 \%$, respectively, and each additional involved lymph node was reported to increase cancer-specific mortality by $5.1 \%$ and overall mortality by $5.6 \%$.

CN can be performed via open or laparoscopic approach. Patients who undergo the laparoscopic procedure generally tend to have shorter hospital stays and can start immunotherapy earlier. Partial nephrectomy can be performed in cases of 
asynchronous bilateral renal tumor. Complication rates may be high depending on tumor size. When compared with radical nephrectomy, nephron-sparing surgery can be considered within the context of $\mathrm{CN}$ in very carefully selected cases. Complications may delay transition to systemic therapy. An increased complication rate has been reported in patients aged $>75$ years, those with low performance status, those with high comorbidities, and those with $\geq 2$ metastases. Mortality rate and hospital experience are both correlated with complication rate. $\mathrm{CN}$ complication rates vary in the literature, but mortality and morbidity rates are higher compared to radical nephrectomy performed for local disease.

$\mathrm{CN}$ combined with systemic therapy is still practiced, while immunotherapy (IFN, IL-2) has been replaced by targeted therapies. The PFS advantage was 11 months in the group that underwent nephrectomy followed by sunitinib versus 6 months in the group treated with sunitinib without nephrectomy (3). Despite the bias in the selection of suitable patients for surgery, the IMDC noted the benefit of $\mathrm{CN}$ in terms of $\mathrm{OS}$ in the era of targeted therapy (31). According to this, patients with poor prognosis and expected survival less than 12 months did not benefit from surgery. In combination therapy research, studies on $\mathrm{CN}$ followed by immunotherapy are older, but studies on $\mathrm{CN}$ followed by targeted therapy are emerging. The role of $\mathrm{CN}$ is still debated in terms of evidence-based medicine. You et al. (32) performed $\mathrm{CN}$ before sunitinib or sorafenib in 45 patients and administered systemic therapy alone to 33 patients. They reported no statistically significant difference between the groups with and without CN in terms of PFS and OS. Gore et al. (33) reported significant improvement in PFS (12 months vs. 6.5 months) in patients who underwent $\mathrm{CN}$ prior to using sunitinib. Choueiri et al. (34) assessed the effect of $\mathrm{CN}$ on patients receiving targeted therapy. They compared patients who underwent CN followed by sunitinib, sorafenib, or bevacizumab therapy with those who received systemic therapy only. In the $\mathrm{CN}$ group, HR was $0.68(p=0.04)$ for $O S$, and the overall response rate was $26.3 \%$ versus $11.5 \%$ in patients without CN. Heng et al. (35) compared patients who underwent $\mathrm{CN}$ prior to systemic therapy, and patients treated with targeted therapy only. The median OS of patients who underwent CN was 20.6 months, significantly longer than the 9.5 months in the group without CN. Hanna et al. (36) evaluated CN + targeted therapy in 5374 patients in the National Cancer Database and targeted therapy alone in 10.016 patients. The risk of mortality was found to be lower in the $\mathrm{CN}$ group (HR 0.45). Young age, being treated at an experienced center, low tumor stage, and clinically negative lymph nodes have been identified as good prognostic factors with CN. In a meta-analysis, Petrelli et al. (37) examined the OS results of $\mathrm{CN}$ and targeted therapy, and reported significantly reduced mortality risk with $\mathrm{CN}(\mathrm{HR} 0.46 ; \mathrm{p}<0.01)$. In the Clinical Trial to Assess the Importance of Nephrectomy (CARMENA) study, mRCC patients were randomized to a sunitinib arm and a $\mathrm{CN}+$ sunitinib arm. CARMENA is a non-inferiority study and upon completion will allow a better estimation of the benefits of $\mathrm{CN}$. The outcomes of this study will elucidate the role of $\mathrm{CN}$ in the era of targeted therapy. Another important study is the Immediate Surgery or Surgery After Sunitinib Malate in Treating Patients with Metastatic Kidney Cancer (SURTIME) study by the EORTC, which is comparing nephrectomy + sunitinib to sunitinib
+ nephrectomy. In the SURTIME study, it will be more difficult to analyze the true value of $\mathrm{CN}$.

\section{Conclusion}

mRCC is a complex disease and carries a poor prognosis. $\mathrm{CN}$, neoadjuvant/adjuvant systemic immunotherapy/targeted therapy and, if necessary, metastasectomy are current complementary approaches that partially extend the survival of patients. Large-scale, randomized prospective studies are needed to explore possibilities such as optimal sequences and combinations of these therapies.

\section{Questions}

1. Which targeted therapeutic agent was not recommended as first-line treatment?

Everolimus.

2. What is the most favorable location for metastasectomy?

The lung.

3. What are two important studies on the combined use of cytoreductive nephrectomy with targeted agents?

The CARMENA and SURTIME studies.

\section{Ethics}

Peer-review: Internally peer-reviewed.

\section{Authorship Contributions}

Surgical and Medical Practices: K.F.N., Concept: K.F.N., Design: K.F.N., Data Collection or Processing: B.Ö., Analysis or Interpretation: B.Ö., Literature Search: B.Ö., Writing: K.F.N..

Conflict of Interest: No conflict of interest was declared by the authors.

Financial Disclosure: The authors declared that this study received no financial support.

\section{References}

1. Janzen NK, Kim HL, Figlin RA, et al. Surveillance after radical or partial nephrectomy for localized renal cell carcinoma and management of recurrent disease. Urol Clin North Am 2003;30:843-852.

2. Motzer RJ, Mazumdar M, Bacik J, et al. Survival and prognostic stratifcation of 670 patients with advanced renal cell carcinoma. J Clin Oncol 1999;17:2530-2540.

3. Motzer RJ, Hutson TE, Tomczak P, et al. Sunitinib versus interferon alfa in metastatic renal-cell carcinoma. N Engl J Med 2007;356:115-124.

4. Motzer RJ, Hutson TE, Cella D, et al. Pazopanib versus sunitinib in metastatic renal-cell carcinoma. N Engl J Med 2013;369:722-731.

5. Escudier B, Eisen T, Stadler WM, et al. TARGET Study Group. Sorafenib in advanced clear-cell renal-cell carcinoma. N Engl J Med 2007;356:125-134.

6. Motzer RJ, Hutson TE, McCann L, et al. Overall survival in renalcell carcinoma with pazopanib versus sunitinib. N Engl J Med 2014; 370:1769-1770.

7. Hudes G, Carducci M, Tomczak P, et al. Temsirolimus, interferon alfa, or both for advanced renal-cell carcinoma. $N$ Engl J Med 2007; 356:2271-2281.

8. Hutson TE, Lesovoy V, Al-Shukri S, et al. Axitinib versus sorafenib as first-line therapy in patients with metastatic renal-cell carcinoma: a randomised open-label phase 3 trial. Lancet Oncol 2013;14:1287-1294.

9. Rini BI, Halabi S, Rosenberg JE, et al. Phase III trial of bevacizumab plus interferon alfa versus interferon alfa monotherapy in patients with metastatic renal cell carcinoma: final results of CALGB 90206. J Clin Oncol 2010;28:2137-2143. 
10. Rini BI, Escudier B, Tomczak P, et al. Comparative effectiveness of axitinib versus sorafenib in advanced renal cell carcinoma (AXIS): a randomised phase 3 trial. Lancet 2011;378:1931-1939.

11. Motzer RJ, Escudier B, Oudard S, et al. Phase 3 trial of everolimus for metastatic renal cell carcinoma: final results and analysis of prognostic factors. Cancer 2010;116:4256-4265.

12. Ravaud A, Barrios CH, Alekseev B, et al. RECORD-2: phase II randomized study of everolimus and bevacizumab versus interferon $\alpha-2 a$ and bevacizumab as firstline therapy in patients with metastatic renal cell carcinoma. Ann Oncol 2015;26:1378-1384.

13. Rini BI, Bellmunt J, Clancy J, et al. Randomized phase III trial of temsirolimus and bevacizumab versus interferonalfa and bevacizumab in metastatic renal cell carcinoma: INTORACT trial. J Clin Oncol 2014;32:752-759.

14. Motzer RJ, Hutson TE, Ren $M$, et al. Independent assessment of lenvatinib plus everolimus in patients withmetastatic renal cell carcinoma. Lancet Oncol 2016;17:e4-e5.

15. Zhou L, Liu XD, Sun $M$, et al. Targeting MET and AXL overcomes resistance to sunitinib therapy in renal cell carcinoma. Oncogene 2016;35:2687-2697.

16. Choueiri TK, Escudier B, Powles T, et al. Cabozantinib versus everolimus in advanced renal cell carcinoma (METEOR):final results from a randomised, openlabel, phase 3 trial. Lancet Oncol 2016;17:917-927.

17. Motzer RJ, Rini Bl, McDermott DF, et al. Nivolumab for metastatic renal cell carcinoma: results of a randomized phasell trial. J Clin Oncol 2015;33:1430-1437.

18. Armstrong AJ, Halabi S, Eisen T, et al. Everolimus versus sunitinib for patients with metastatic non-clear cell renal cellcarcinoma (ASPEN): a multicentre, openlabel, randomised phase 2 trial. Lancet Oncol 2016;17:378-388.

19. Choueiri TK, Halabi S, Sanford BL, et al. Cabozantinib versus sunitinib (CABOSUN) as initial targeted therapy for patients with metastatic renal cell carcinoma of poor or intermediate risk: the Alliance A031203 CABOSUN trial. J Clin Oncol 2017;35:591-597.

20. Toni K, Choueiri MD, Robert J, Motzer MD. Systemic Therapy for Metastatic Renal Carcinoma. N Engl J Med 2017;376:354-366.

21. Naito S, Yamamoto N, Takayama T, et al. Prognosis of Japanese metastatic renal cell carcinoma patients in the cytokine era: a cooperative group report of 1463 patients. Eur Urol 2010;57:317-325.

22. Kavolius JP, Mastorakos DP, Pavlovich C, et al. Resection of metastatic renal cell carcinoma. J Clin Oncol 1998;16:2261-2266.

23. Dabestani S, Marconi L, Hofmann F, et al. Local treatments for metastases of renal cell carcinoma: a systematic review. Lancet Oncol 2014; 15:e549-e561.
24. Karam JA, Rini BI, Varella L, et al. Metastasectomy after targeted therapy in patients with advanced renal cell carcinoma. J Urol 2011;185:439-444.

25. Ikushima $H$, Tokuuye $K$, Sumi $M$, et al. Fractionated stereotactic radiotherapy of brain metastases from renal cell carcinoma. Int J Radiat Oncol Biol Phys 2000;48:1389-1393.

26. Thomas AZ, Adibi M, Borregales LD, et al. Role of metastasectomy in metastatic renal cell carcinoma. Curr Opin Urol 2015;25:381-389.

27. Flanigan RC, Salmon SE, Blumenstein BA, et al. Nephrectomy followed by interferon alfa- $2 \mathrm{~b}$ compared with interferon alfa- $2 \mathrm{~b}$ alone for metastatic renal-cell cancer. N Engl J Med 2001;345:1655-1659.

28. Mickisch GH, Garin A, van Poppel H, et al. Radical nephrectomy plus interferon-alfa-based immunotherapy compared with interferon alfa alone in metastatic renal-cell carcinoma: a randomised trial. Lancet 2001;358:966-970.

29. Hanna N, Sun M, Meyer CP, et al. Survival analyses of patients with metastatic renal cancer treated with targeted therapy with or without cytoreductive nephrectomy: a National Cancer Data Base study. J Clin Oncol 2016;34:3267-3275.

30. Trinh QD, Sukumar S, Schmitges ], et al. Effect of nodal metastases on cancer-specific mortality after cytoreductive nephrectomy. Ann Surg Oncol 2013;20:2096-2102.

31. Heng DY, Wells JC, Rini BI, et al. Cytoreductive nephrectomy in patients with synchronous metastases from renal cell carcinoma: results from the International Metastatic Renal Cell Carcinoma Database Consortium. Eur Urol 2014;66:704-710.

32. You $\mathrm{D}$, Jeong IG, Ahn JH, et al. Te value of cytoreductive nephrectomy for metastatic renal cell carcinoma in the era of targeted therapy. ] Urol 2011;185:54-59.

33. Gore ME, Szczylik C, Porta C, et al. Final results from the large sunitinib global expanded-access trial in metastatic renal cell carcinoma. $\mathrm{Br}$ J Cancer 2015;113:12-19.

34. Choueiri TK, Xie W, Kollmannsberger C, et al. Te impact of cytoreductive nephrectomy on survival of patients with metastatic renal cell carcinoma receiving vascular endothelial growth factor targeted therapy. J Urol 2011;185:60-66.

35. Heng DY, Wells JC, Rini BI, et al. Cytoreductive nephrectomy in patients with synchronous metastases from renal cell carcinoma: results from the International Metastatic Renal Cell Carcinoma Database Consortium. Eur Urol 2014;66:704-710.

36. Hanna N, Sun M, Meyer CP, et al. Survival analyses of metastatic renal cancer patients treated with targeted therapy with or without cytoreductive nephrectomy: a National Cancer Data Base Study. J Clin Oncol 2016;34:3267-3275.

37. Petrelli F, Coinu A, Vavassori I, et al. Cytoreductive nephrectomy in metastatic renal cell carcinoma treated with targeted therapies: a systematic review with a meta-analysis. Clin Genitourin Cancer 2016;14:465-447. 